Check for updates

Cite this: Mater. Chem. Front., 2020, 4, 1773

Received 13th March 2020,

Accepted 22nd April 2020

DOI: 10.1039/d0qm00154f

rsc.li/frontiers-materials

\section{AlEgen based polymorphs with solvent regulated crystal-to-crystal switch properties $\dagger$}

\author{
Liming Lai, ${ }^{a}$ Bing Fang, ${ }^{a}$ Wenyu Cheng, ${ }^{a}$ Pengyu Li, ${ }^{a}$ Yantu Zhang ${ }^{b}$ and \\ Meizhen Yin (iD) *a
}

\begin{abstract}
Organic polymorphic materials have attracted considerable interest in recent years owing to their unique photophysical properties and application potential in fluorescent sensors and information storage. However, the preparation of organic polymorphs with crystal-to-crystal switch properties remains challenging, which hampers understanding the relationship between molecular stacking and emission properties. Herein, an AIEgen with tunable polymorphism-dependent emission and solvent regulated crystal-to-crystal switch properties is reported. Two polymorphs, an orange crystal (OC) and a red crystal (RC), of the AlEgen with different emission were obtained by slow evaporation in cultivation solvents (dichloromethane (DCM)/n-hexane) with different compositions. The polymorphs can be reversibly switched when exposed to different solvent vapors. Further investigation of the single crystal structures suggests that the molecular stacking mode of the crystal plays an important role in the emission properties. The real-time in situ monitoring of the polymorphs with fluorescence spectra and micrographs when exposed to solvent vapor reveals that the capture/release of DCM molecules induces molecular re-arrangements, and thus causes the switch. Such a conclusion is further supported by thermal recovery experiments. Our study on the crystal-to-crystal switch properties of AlEgen based polymorphs has provided insight into the relationship between molecular stacking and emission properties.
\end{abstract}

\section{Introduction}

Organic luminescent materials are highly attractive materials due to their excellent photophysical properties and practical application potential in fluorescent (FL) sensors, ${ }^{1}$ information anti-counterfeiting and microlasers. ${ }^{2}$ The traditional organic fluorophores easily form strong intermolecular $\pi-\pi$ stacking interactions, and thus are quenched in the aggregation state. ${ }^{3}$ However, Tang and co-workers discovered compounds with aggregation-induced emission (AIE) properties caused by the restriction of intramolecular motion. ${ }^{4}$ The discovery overcomes the aggregation-caused quenching problem of the traditional materials, ${ }^{5}$ and has promoted the development of organic luminescent solid-state materials. ${ }^{6}$ However, the relationship

\footnotetext{
${ }^{a}$ State Key Laboratory of Chemical Resource Engineering, Beijing Advanced Innovation Center for Soft Matter Science and Engineering, Beijing Laboratory of Biomedical Materials, Beijing University of Chemical Technology, Beijing 100029, P. R. China. E-mail: yinmz@mail.buct.edu.cn

${ }^{b}$ College of Chemistry and Chemical Engineering, Yan'an University, Yan'an, Shanxi Province 716000 , P. R. China

$\dagger$ Electronic supplementary information (ESI) available: Additional experimental data, including details of the synthesis, structural information for the compounds $\left({ }^{1} \mathrm{H}\right.$ NMR, ${ }^{13} \mathrm{C}$ NMR and mass spectra), and crystal data. Fig. S1-S20 and Tables S1-S3. CCDC 1973733 and 1973734. For ESI and crystallographic data in CIF or other electronic format see DOI: 10.1039/d0qm00154f
}

between the molecular stacking and the emission properties of solid-state materials still lacks sufficient study.

Crystalline materials show ordered molecular stacking with specific molecular conformations and intermolecular interactions. ${ }^{7}$ The polymorphs of a crystal in different solid-state forms can exhibit completely different emission properties. ${ }^{8}$ Therefore, they have been used as ideal models to study the relationships between molecular stacking and emission properties. ${ }^{9}$ Tang et al. prepared two polymorphs of chalcone derivatives with obvious stacking-dependent emission properties. ${ }^{10}$ Yang et al. reported polymorphs with tunable emission colors upon external stimuli including grinding, heating, and solvent vapor. ${ }^{11}$ However, these polymorphs collapse easily under the external stimuli and thus fail the crystal-to-crystal switch test, which makes the study of the structure-property relationships challenging.

Herein, we designed and synthesized a compound, DCzFO, with intramolecular charge transfer (ICT) and AIE properties. Two polymorphs of DCzFO, an orange crystal (OC) and a red crystal (RC), with completely different emission were obtained by slow evaporation in solvents with different compositions and their single crystal structures were characterized to explore the relationship between their molecular stacking and emission properties. The results suggest that $\mathbf{R C}$ is compactly stacked due to the more planar molecular conformations and stronger intermolecular interactions, and thus exhibits $74 \mathrm{~nm}$ 
red-shifted emission as compared with that of OC. The realtime in situ monitoring of the polymorph exposed to solvent vapor with micrographs and FL spectra suggests that OC and RC can be reversibly switched when exposed to different solvent vapors. Further investigation suggests that the molecular re-arrangement during the crystal-to-crystal switch is caused by the capture/release of dichloromethane (DCM) molecules. Our work would help to understand the relationship between the molecular stacking and the emission properties of polymorphs.

\section{Results and discussion}

\subsection{Design and synthesis of DCzFO}

Scheme 1 shows the synthesis route to DCzFO. The compound was prepared by a one-step reaction between 2,7-dibromo-9fluorenone and $9 \mathrm{H}$-carbazole. It is designed as a donor-accepterdonor molecule, where a fluorenone unit functions as the electron-acceptor. The carbazole group with strong electrondonating capability serves as the electron donor. ${ }^{12}$ ICT occurs from the carbazole groups to the fluorenone moiety. The good molecule planarities of the fluorenone and carbazole units are conducive to the assembly of well-ordered structures. The chemical structure of the compound is confirmed by ${ }^{1} \mathrm{H}$ NMR, ${ }^{13} \mathrm{C}$ NMR, ESI-TOF and X-ray single-crystal structural analysis. The detailed synthesis procedure and characterization can be found in the $\mathrm{ESI} \dagger$ (Fig. S1-S3).

\subsection{ICT and AIE properties of DCzFO}

The photophysical properties of DCzFO were investigated in different polarity solvents. As shown in Fig. 1a, DCzFO exhibits two absorption bands centered at $340 \mathrm{~nm}$ and $460 \mathrm{~nm}$, which are respectively due to the $\pi-\pi^{*}$ transition and the ICT from the electron-donating carbazole units to the electron-accepting fluorenone unit. $^{13}$ Altering the solvent polarity shows no significant effect on the absorption spectra of DCzFO (Fig. 1a) but affects its FL emission remarkably (Fig. 1b). With the increase of the solvent polarity, the FL emission peak of DCzFO gradually red-shifts from $543 \mathrm{~nm}$ to $602 \mathrm{~nm} .{ }^{10}$ The electronic structure of DCzFO was then determined by density functional theory calculations to better understand its ICT feature (Fig. 1c). As expected, the highest occupied molecular orbital is located over the whole molecule and the lowest unoccupied molecular orbital is mainly found on the fluorenone unit, indicating the ICT feature of DCzFO. ${ }^{8 a, 13}$

The AIE properties of DCzFO were then investigated. DMSO is a good solvent of the compound and water is a poor solvent. It is not emissive in the good solvent. Therefore, the emission behaviors of DCzFO aggregates were investigated in $\mathrm{DMSO} / \mathrm{H}_{2} \mathrm{O}$ solutions with different water fractions $\left(f_{\mathrm{w}}\right)$. The FL emission

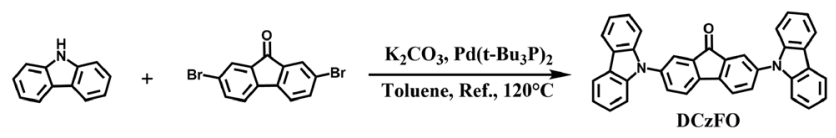

Scheme 1 Synthetic route to and the structure of DCzFO.
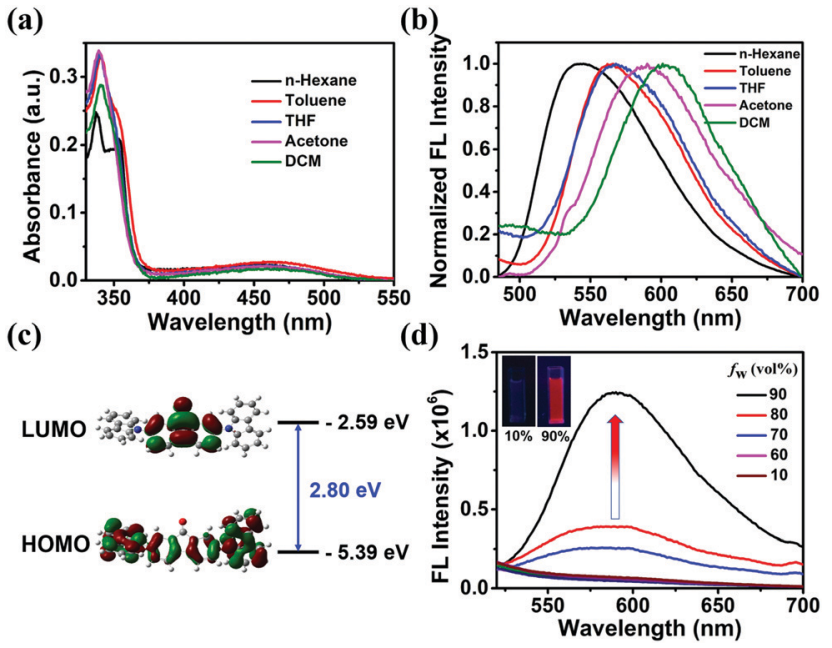

Fig. 1 (a) UV-vis and (b) normalized fluorescence (FL) spectra $\left(\lambda_{\text {ex }}=\right.$ $365 \mathrm{~nm})$ of DCzFO $(10 \mu \mathrm{M})$ in different solvents. (c) Optimized geometry and calculated HOMO and LUMO levels for DCzFO. (d) FL spectra $\left(\lambda_{\text {ex }}=\right.$ $365 \mathrm{~nm})$ of DCzFO $(10 \mu \mathrm{M})$ in $\mathrm{DMSO} / \mathrm{H}_{2} \mathrm{O}$ mixtures with different water fractions $\left(f_{\mathrm{w}}\right)$. Inset: $\mathrm{FL}$ images of $\mathrm{DCzFO}(10 \mu \mathrm{M})$ in $\mathrm{DMSO} / \mathrm{H}_{2} \mathrm{O}$ mixtures with $f_{\mathrm{w}}=0 \%$ and $90 \%$ under irradiation with $365 \mathrm{~nm}$ UV light.

intensity changed slightly as $f_{\mathrm{w}}$ varied in the range $10-60 \%$ and increased dramatically as $f_{\mathrm{w}}$ increased to $70 \%$. DCzFO exhibits 26-fold stronger FL emission intensity in the $\mathrm{DMSO} / \mathrm{H}_{2} \mathrm{O}$ mixture with an $f_{\mathrm{w}}$ of $90 \%$ than in pure DMSO, showing typical AIE characteristics (Fig. 1d). The AIE properties of DCzFO can result in strong solid-state FL emission. ${ }^{14}$

\subsection{Solid-state emission and crystal structure of DCzFO}

The as-prepared DCzFO shows a yellow emission peak at $575 \mathrm{~nm}$ (Fig. S4, ESI $\dagger$ ). The rigid planar structure of the fluorenone and carbazole units endows DCzFO with outstanding crystallization capacity. A rod-like orange crystal (OC) of DCzFO was obtained by slow evaporation under ambient conditions in a DCM/ $n$-hexane mixture with $\mathrm{v}: \mathrm{v}=1: 3$. Increasing the volume ratio of DCM in the DCM $/ n$-hexane mixture to $\mathrm{v}: \mathrm{v}=1: 1$ results in a needle-like red crystal (RC). The main peaks of the ${ }^{1} \mathrm{H}$ NMR spectra of $\mathbf{O C}$ are similar to those of $\mathbf{R C}$, indicating the polymorphism property of DCzFO (Fig. S5, ESI $\dagger$ ). However, the polymorphs exhibit different morphologies and FL colors (Fig. 2a, insets). As shown in Table 1 and Fig. 2c, OC exhibits orange FL emission peaking at $581 \mathrm{~nm}$ with a quantum yield of $\sim 19.3 \%$. RC displays red FL emission peaking at $655 \mathrm{~nm}$ $\left(\Phi_{\mathrm{F}}=4.3 \%\right)$. The FL color was determined by the Commission Internationale de l'Éclairage 1931 coordinates (Fig. S6a, ESI $\dagger$ ). Compared with that of $\mathbf{O C}$, the absorption band of $\mathbf{R C}$ is broader and red-shifted. In addition, the nonradiative rate of $\mathbf{R C}$ is 6-fold higher than that of OC. Therefore, it is speculated that more energy from the excitation of $\mathbf{R C}$ is lost during the nonradiative process, resulting in the weaker FL intensity and lower quantum yield. ${ }^{10}$ Such vastly different solid-state emission properties of OC and RC may be ascribed to their different stacking modes. ${ }^{11,14 c}$ To confirm the hypothesis, the stacking modes of OC and $\mathbf{R C}$ were investigated by powder X-ray diffraction (PXRD). 
(a)
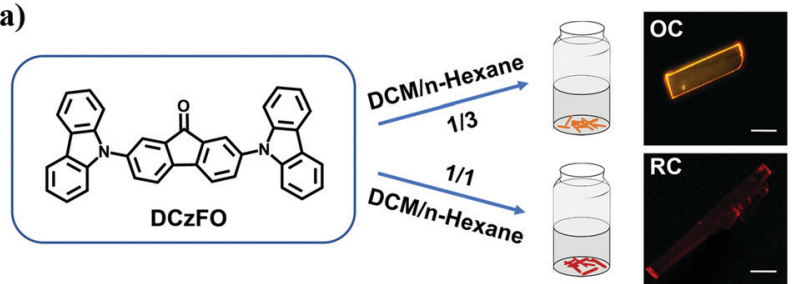

(b)

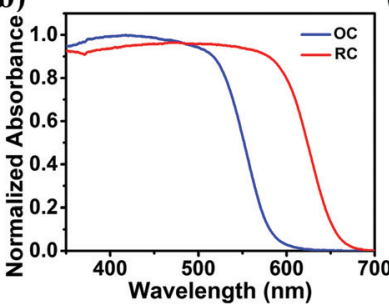

(c)

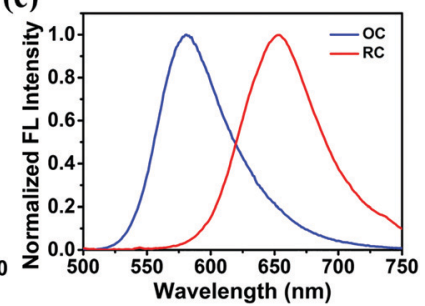

Fig. 2 (a) Schematic illustration of the preparation process of the polymorphs and the FL micrographs of $\mathrm{OC}$ and RC crystals irradiated under a UV lamp (365 nm). (b) Normalized UV-vis and (c) FL spectra $\left(\lambda_{\text {ex }}=400 \mathrm{~nm}\right)$ of OC (blue line) and RC (red line). Scale bar: $200 \mu \mathrm{m}$.

Table 1 The photophysical parameters of $\mathbf{O C}$ and $\mathbf{R C}$ including emission maxima $\left(\lambda_{\mathrm{FL}}\right)$, quantum yields $\left(\Phi_{\mathrm{F}}\right)$, FL lifetimes $\left(\tau_{\mathrm{FL}}\right)$, radiative rates $\left(k_{\mathrm{r}}\right)$, and non-radiative rates $\left(k_{\mathrm{nr}}\right)$ of crystals, $k_{\mathrm{r}}=\Phi_{\mathrm{F}} / \tau_{\mathrm{FL}} ; k_{\mathrm{nr}}=\left(1-\Phi_{\mathrm{F}}\right) / \tau_{\mathrm{FL}}$

\begin{tabular}{lllllc}
\hline Crystal & $\lambda_{\mathrm{FL}}(\mathrm{nm})$ & $\tau_{\mathrm{FL}}(\mathrm{ns})$ & $\Phi_{\mathrm{F}}(\%)$ & $k_{\mathrm{r}}\left(10^{7} \mathrm{~s}^{-1}\right)$ & $k_{\mathrm{nr}}\left(10^{7} \mathrm{~s}^{-1}\right)$ \\
\hline OC & 581 & 8.6 & 19.3 & 2.2 & 9.1 \\
RC & 655 & 1.7 & 4.3 & 2.5 & 55.0
\end{tabular}

There are more sharp peaks in the PXRD pattern of RC at the lower end of 2 theta, as compared with that of OC, which indicates well-ordered crystal structure and different molecular stacking modes of OC and RC (Fig. S7, ESI $\dagger$ ).

To further understand the solid-state emission behaviors, the crystal structures of the two polymorphs were determined by X-ray single-crystal structure analysis. The results are summarized in Table S3 (ESI $\dagger$ ). OC is a crystal system of monoclinic space group $P 22_{1} / c$ with unit cell parameters of $a=19.8760(19) \AA$, $b=7.9623(9) \AA$ and $c=17.0139(19) \AA$. Its unit cell contains four DCzFO molecules (Fig. S8a, ESI $\dagger$ ). The twisted conformation of each molecule with the large dihedral angle between the fluorenone moiety and carbazole rings results in a nearly vertical position relative to the carbazole units on the two end sides (Fig. 3a and c). Therefore, the DCzFO molecules are conjugated in a head-to-tail staggered molecular stacking mode along the $a$-axis direction in OC (Fig. S9, ESI $\dagger$ ). Weak $\pi-\pi$ interactions (4.036-4.111 $\AA$ ) and low overlap degrees of the fluorenone conjugate planes are also observed in OC, which is attributed to the twisted molecular conformation of DCzFO and large steric hindrance (Fig. S9c, ESI $\dagger$ ). Each molecule connects to its adjacent molecules by next-nearest-neighbor intermolecular $\mathrm{H}$-bonds $(\mathrm{C}-\mathrm{H} \cdots \mathrm{O})$ with a length of $2.528 \AA$, nonclassical intermolecular H-bonds $(\mathrm{C}-\mathrm{H} \cdots \mathrm{N})$ with a length of $3.443 \AA$ and weak $\mathrm{C}-\mathrm{H} \cdots \pi$ interactions with lengths of $2.929 \AA$ and $3.571 \AA$. No obvious $\pi-\pi$ interaction is observed (Fig. S9b, ESI $\dagger$ ). The $\mathrm{C}-\mathrm{H} \cdots \mathrm{O}$ hydrogen bond $(2.528 \AA$ ) between the $\mathrm{O}$ atom of the
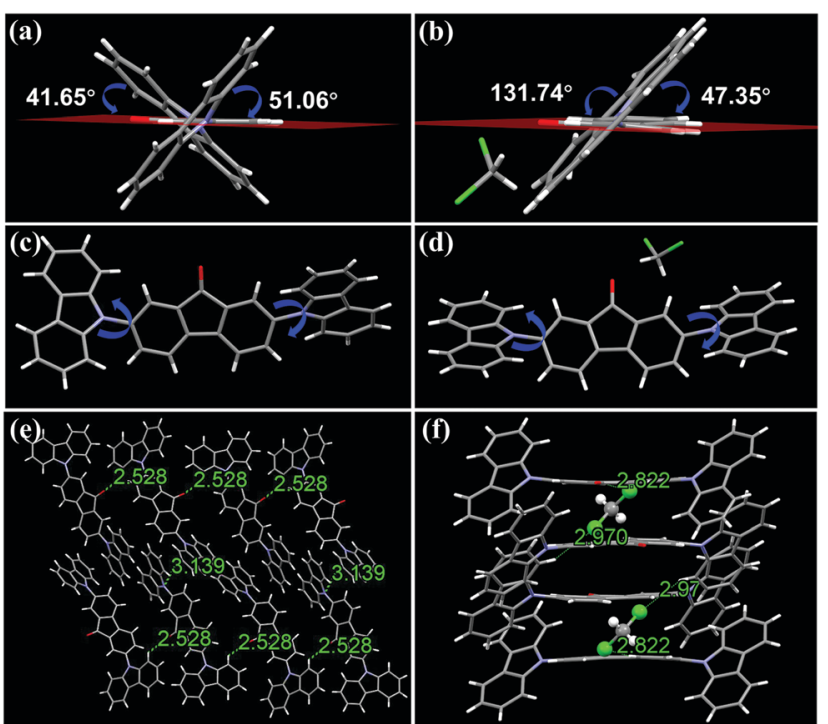

(g)
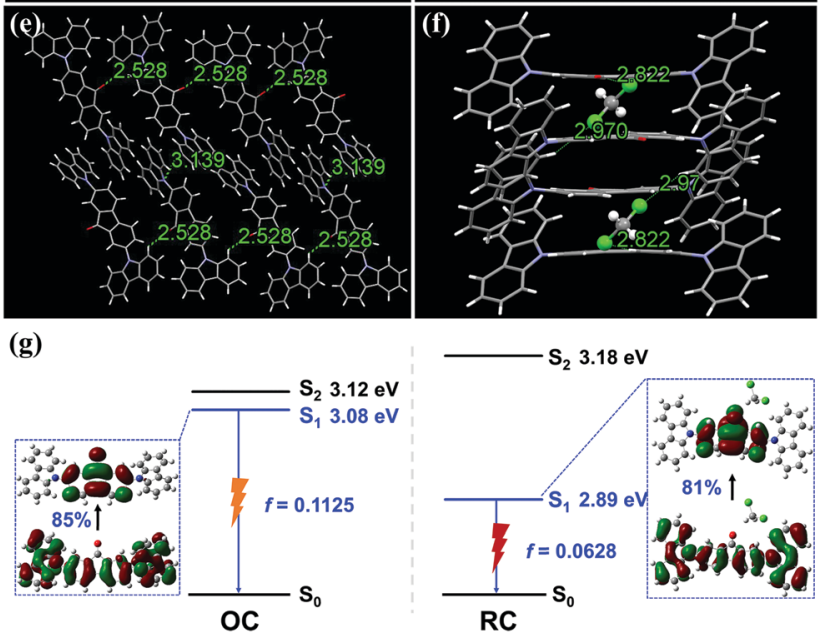

Fig. 3 Dihedral angle of DCzFO in (a) $\mathbf{O C}$ and (b) RC crystals. Twisted molecular conformation of DCzFO in (c) OC and (d) RC crystals. (e) Intermolecular interactions between adjacent molecules of the $\mathbf{O C}$ crystal. (f) Two adjacent dimers and intermolecular interactions of DCzFO and DCM in the RC crystal. (g) Theoretically calculated vertical excitation energies, transition natures of the excited states and oscillator strengths (f) for OC and RC. Interaction distances (dashed lines) are in $\AA$.

fluorenone unit and the $\mathrm{H}$ atom of the carbazole ring links adjacent DCzFO molecules to form a loose 3-dimensional net structure (Fig. 3e).

RC is formed in a different molecular stacking mode from the head-to-tail staggered molecular stacking mode for OC. It is also a monoclinic crystal system, but belongs to the space group $C 2 / c$ with unit cell parameters of $a=35.988(2) \AA$, $b=10.9785(6) \AA$, and $c=13.4888(10) \AA$. There are eight DCzFO molecules in the unit cell, which are parallel to each other in a slightly staggered orientation. Therefore, the molecular conformation of DCzFO in RC is more planar than that in OC (Fig. 3b and d). It is worth noting that four DCM molecules are detected in the crystal lattice of RC (Fig. S8b, ESI $\dagger$ ), suggesting that it plays a key role in the molecular stacking of RC. Strong

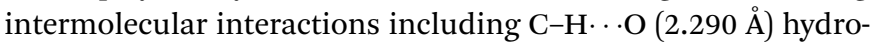
gen bonds and $\mathrm{C}-\mathrm{H} \cdots \mathrm{Cl}(2.822 \AA)$ halogen bonds are found between DCzFO and DCM molecules, which restrict the molecular conformation of DCzFO. The two carbazoles on both ends are almost horizontal. The planar molecular conformation increases the molecular overlap and electronic transition, which causes larger electronic excited-state delocalization as compared with that of $\mathbf{O C}$. The crystal density of $\mathbf{R C}$ is measured to be $1.385 \mathrm{mg} \mathrm{mm}^{-3}$, higher than that of OC (Table S3, ESI $\dagger$ ). 
The molecules in RC completely overlap with each other along the long molecular axis, and thus form a tightly stacked structure via strong $\pi-\pi$ interactions (3.446 $\AA$ ). Along the crystallographic $c$-axis, a typical step-like stacking structure is observed (Fig. 3f). The DCM molecule serves as a linker to connect two adjacent molecules tightly via $\mathrm{C}-\mathrm{H} \cdots \mathrm{Cl}(2.822 \AA)$ and $\mathrm{C}-\mathrm{H} \cdots \mathrm{O}(2.290 \AA)$ hydrogen bonds, which results in "dimers". The "dimers" are antiparallel to each other with a small slip angle of $24.54^{\circ}$ along the $b$-axis. The distance between the adjacent "dimers" is only $~ 3.566 \AA$ (Fig. S10b, ESI $\dagger$ ). Such a short distance is indicative of strong $\pi-\pi$ stacking. Therefore, the "dimers" are packed to form an infinite 3-dimensional staggered space network (Fig. S10c, ESI $\dagger$ ). The orderly stacked structure of the single crystal in the preferred orientation immensely increases the degree of conjugation. ${ }^{10}$

To further discover the emission properties of $\mathbf{O C}$ and $\mathbf{R C}$, theoretical calculations were performed through the hybrid quantum mechanics and molecular mechanics (QM/MM) approach (Fig. S11, ESI $\dagger$ ). The calculations indicate that the molecular conformation has a significant influence on the excited state properties of DCzFO, as shown in Fig. $3 \mathrm{~g}$. The OC conformer has a higher $\mathrm{S}_{1}$ energy level $(3.08 \mathrm{eV})$ than RC $(2.89 \mathrm{eV})$, resulting in a red-shift in the FL spectra. In addition, the change of the molecular conformation affects the oscillator strength $(f)$ of the $\mathrm{S}_{1}-\mathrm{S}_{0}$ transition. With the dihedral angle of the two carbazole groups decreasing, the $f$ value reduces from 0.1125 to 0.0628 , suggesting a lower $\Phi_{\mathrm{F}}$ value and stronger emission for the $\mathbf{R C}$ crystal. ${ }^{15}$

Based on these results, the relationship between the emission properties of DCzFO and its stacking mode can be concluded as follows (Fig. S12, ESI $\dagger$ ). DCzFO can exhibit emission in a wide range from $\lambda_{\mathrm{em}}=575 \mathrm{~nm}$ (original powder) to $\lambda_{\mathrm{em}}=655 \mathrm{~nm}(\mathbf{R C})$. Each emission state corresponds to a distinct molecular stacking mode. The original DCzFO powder is in the monomeric state with negligible intermolecular interactions and thus exhibits no molecular stacking. Therefore, the emission properties of DCzFO in the solid state are influenced by the molecular stacking mode, which can be regulated by the molecular conformation and intermolecular interactions. ${ }^{16}$ The more planar molecular conformation and stronger intermolecular interactions result in the more compact stacking structure of RC with dramatically redshifted emission and weaker FL intensity.

\subsection{Solvent vapor regulated crystal-to-crystal switch}

The emission properties of solid-state materials can be altered with external stimuli, such as solvent vapor, heating, and grinding. ${ }^{17}$ When OC is exposed to DCM vapor, the emission peak of the sample (OC-fumed) red-shifts to $654 \mathrm{~nm}$, accompanied by an obvious FL color change from orange to red. OC-fumed exhibits a similar FL spectrum to that of $\mathrm{RC}\left(\lambda_{\mathrm{em}}=655 \mathrm{~nm}\right)$, and, vice versa, the RC sample fumed with $n$-hexane vapor (RC-fumed, $\lambda_{\mathrm{em}}=579 \mathrm{~nm}$ ) exhibits a similar FL emission peak to that of OC $\left(\lambda_{\mathrm{em}}=581 \mathrm{~nm}\right)$ (Fig. 4a and b). The transitions can be repeated for at least three cycles without variation in intensity (Fig. 4c). In addition, both OC-fumed and RC-fumed exhibit strong diffraction peaks, showing that the fumed samples maintain the integrity of the corresponding crystal structure (Fig. 4d). The diffraction (a)

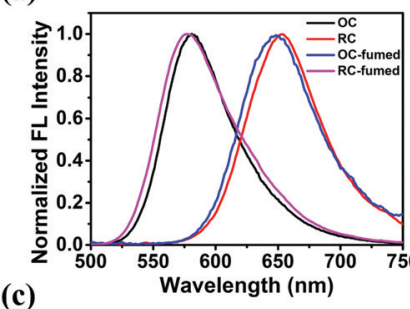

(b)

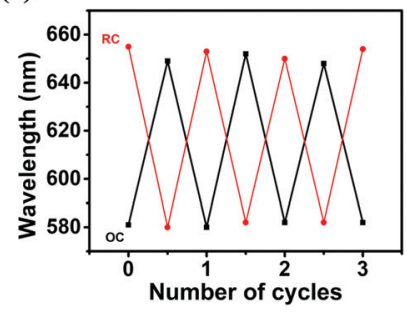

(d)

Fig. 4 (a) Normalized FL spectra ( $\lambda_{\mathrm{ex}}=400 \mathrm{~nm}$ ) of the original $\mathrm{OC}$ and RC and the corresponding fumed samples, OC-fumed and RC-fumed, under excitation at $365 \mathrm{~nm}$. (b) FL images of the crystals before and after vapor fuming. (c) Cyclic FL emission of the crystal-to-crystal switch. (d) Powder diffraction pattern of the original and fumed crystals.

pattern of OC-fumed is in good agreement with that of RC, and the diffraction pattern of RC-fumed matches that of OC well, indicating that the solvent vapor can directly induce a crystal-to-crystal switch between OC and RC.

To better understand the crystal-to-crystal switch, the process was monitored in situ under a microscope upon the FL emission change during the vapor fuming. An OC sample fixed on a quartz plate was put in a Petri dish containing DCM vapor (Fig. 5a). The color and the FL emission spectra of the sample were recorded every $5 \mathrm{~min}$. It is found that the color of the crystal began to change from both end sides when fumed for $5 \mathrm{~min}$. The main body of the crystal gradually exhibits a red FL color as the fuming time is prolonged from $5 \mathrm{~min}$ to $50 \mathrm{~min}$. The entire crystal shows similar red emission to RC in $60 \mathrm{~min}$ (Fig. 5a). The spectra recorded during the fuming clearly reveal an intermediate state during the crystal-to-crystal switch in the DCM vapor, accompanied by changes of the emission wavelength and intensity (Fig. 5b). The emission switch of RC to OC is observed upon exposure to $n$-hexane vapor in $3 \mathrm{~h}$ (Fig. $5 \mathrm{c}$ ). The detailed results of the variations in the FL spectra and FL intensity with the fuming time can be found in Fig. S13 (ESI $\dagger$ ). These real-time observations further indicate that the solvent vapor can induce a crystal-to-crystal switch between OC and RC.

As demonstrated above, DCzFO molecules are loosely stacked in the OC crystal. In contrast, RC is more compactly stacked, and DCM molecules are found in its crystal lattices. Therefore, the release/inclusion of DCM molecules may play a key role in the switch between OC and RC. When OC is exposed to DCM vapor, the DCM molecules can enter its loose lattices and interact with DCzFO molecules. The robust intermolecular interactions between DCzFO and DCM inhibit the structure conformation, leading to the crystal-to-crystal switch from OC to RC. The hypothesis is confirmed by ${ }^{1} \mathrm{H}$ NMR analyses of the samples before and after vapor treatment (Fig. S14, ESI $\dagger$ ). 
(a)
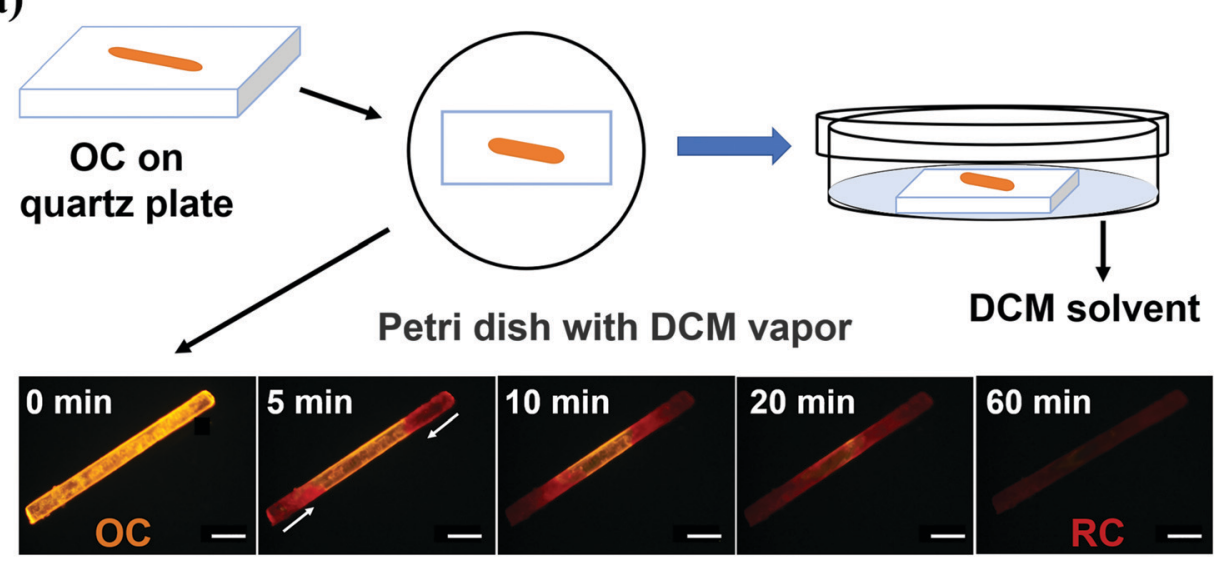

(b)

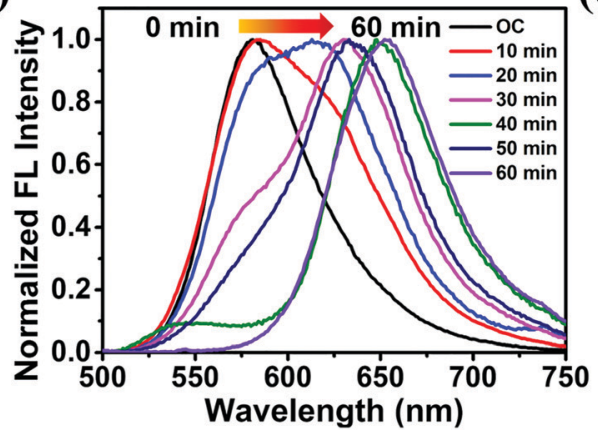

(c)

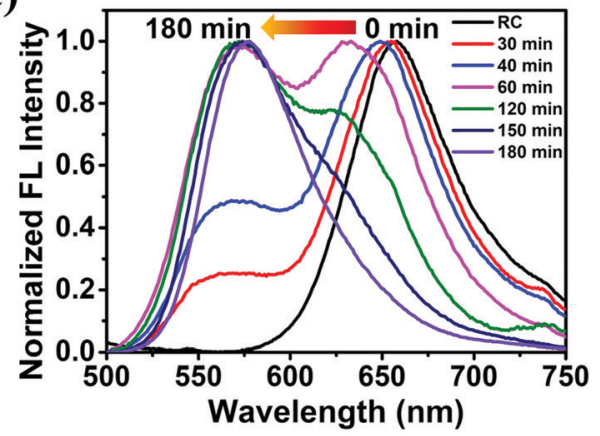

Fig. 5 (a) Schematic vapor fuming process of $\mathbf{O C}$ in a Petri dish with DCM vapor and micrographs showing the crystal-to-crystal switching process upon vapor fuming under UV irradiation (365 nm). (b) Normalized FL spectra ( $\lambda_{\text {ex }}=400 \mathrm{~nm}$ ) of OC at 10 minute intervals from 0 to $60 \mathrm{~min}$. (c) Normalized FL spectra $\left(\lambda_{\text {ex }}=400 \mathrm{~nm}\right)$ of RC at 10 minute fuming time intervals from 0 to $180 \mathrm{~min}$. Scale bar: $200 \mu \mathrm{m}$.

The OC sample fumed with DCM vapor exhibits ${ }^{1} \mathrm{H}$ NMR peaks of the DCM solvent, implying that DCM molecules enter OC lattices to form a co-crystal. The different DCM peak intensity of OC-fumed and RC is ascribed to the capacity of trapping DCM of the single crystal incubation and the fuming process. In contrast, the DCM peaks of the RC-fumed sample are very weak or even disappear. Therefore, the crystal-to-crystal switch from RC to OC is ascribed to the removal of the DCM molecules in $\mathbf{R C}$.

In addition to solvent vapor, heating can induce the switch from RC to OC. The heated RC sample (RC-h) exhibits a similar FL emission spectrum to that of OC, accompanied by increased FL intensity (Fig. S15, ESI $\dagger$ ). ${ }^{1} \mathrm{H}$ NMR analyses also suggest the absence of DCM in RC-h (Fig. S16, ESI $\dagger$ ). The removal of DCM enhances the movement of molecules, which induces the re-arrangement of molecules back to the stacking structure of OC. Based on these observations, it can be concluded that removing the solvent molecules from the crystalline lattice causes molecular re-arrangement, and eventually leads to the crystal-to-crystal switch from RC to OC upon solvent vapor fuming and heating (Fig. S17, ESI $\dagger$ ).

\subsection{Distinct mechanochromic luminescence properties of OC and RC}

The emission properties of a crystal can be tuned by adjusting its stacking modes through external force stimuli. The emission peak of OC red-shifts from $581 \mathrm{~nm}$ to $597 \mathrm{~nm}$ with reduced $\mathrm{FL}$ intensity as it is ground with a pestle (OC-G). In contrast, the ground sample of RC (RC-G) exhibits blue-shifted emission from $655 \mathrm{~nm}$ to $630 \mathrm{~nm}$ with stronger FL intensity (Fig. 6). The detailed photophysical properties of the ground samples can be found in Table S2 and Fig. S18 (ESI $\dagger$ ). Similar to the bulk crystals, the ground samples also exhibit reversible emission switch behaviors when exposed to solvent vapor (Fig. S19, ESI $\dagger$ ). These results suggest that the modulation of molecular stacking involves changes of molecular conformations and intermolecular interactions.

The distinctive mechanochromic luminescence (ML) properties of $\mathbf{O C}$ and $\mathbf{R C}$ are due to their different molecular conformations and intermolecular interactions. OC is loosely stacked with twisted conformations via only weak $\mathrm{C}-\mathrm{N} \cdots \pi$ interactions. The weak intermolecular interactions and loose crystalline lattice are easily destroyed when subjected to external forces, which explains its red-shifted ML. ${ }^{18}$ RC is more tightly stacked via multiple intermolecular interactions. However, the intermolecular interactions between DCM and DCzFO can be destroyed by external force, which breaks the "dimers" and strong molecule conjugation, and thus causes blueshifted ML emission. ${ }^{8 a}$ In addition, the destruction of the close crystalline lattices also removes DCM to some extent, which explains the reversible emission behaviors of the OC-G and RC-G samples. 
(a)

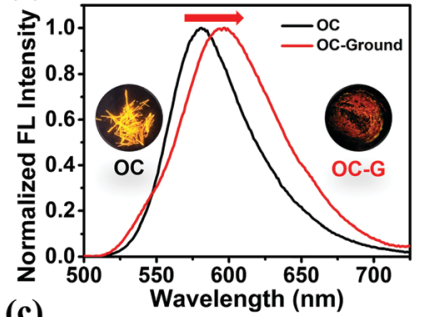

(c)

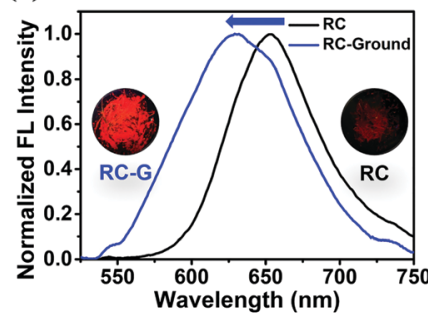

(b)

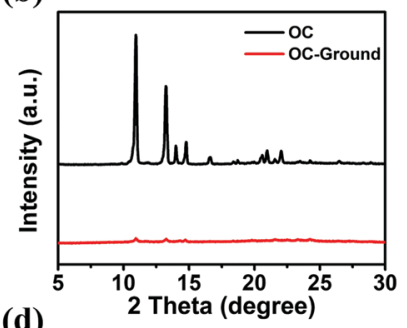

(d)

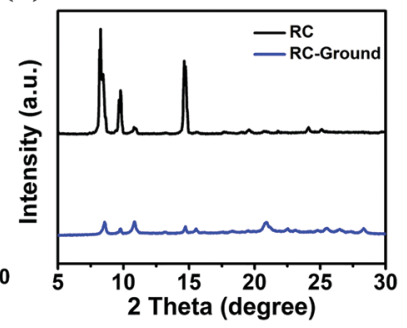

Fig. 6 (a) Normalized FL spectra $\left(\lambda_{\mathrm{ex}}=400 \mathrm{~nm}\right.$ ) and (b) PXRD patterns of OC before and after being ground. (c) Normalized FL spectra $\left(\lambda_{\text {ex }}=\right.$ $400 \mathrm{~nm}$ ) and (d) PXRD patterns of RC before and after being ground.

The multiple stimuli-responsive behaviors of the polymorphs are summarized in Fig. 7a. Inspired by its solvent induced crystalto-crystal switch properties, the application potential of DCzFO is demonstrated in data encryption under UV light irradiation. The compound 3,4,9,10-perylenetetracarboxylic dianhydride (PDA) exhibits similar FL properties (Fig. S20, ESI $\dagger$ ). As shown in Fig. 7b, a digit pattern "888" with a weak red FL color is achieved with RC and PDA powder under UV light irradiation. The color of the RC in the pattern changes from red to orange accompanied by enhanced luminescence intensity when fumed with $n$-hexane vapor. In constrast, the color of the PDA powder in the pattern shows no obvious change when fumed with $n$-hexane vapor. As a result, orange digits "567" appear under UV light irradiation. The pattern " 567 " is successfully restored to the original pattern " 888 " upon fuming with DCM vapor. Therefore, DCzFO has great application potential as a candidate material for data encryption and security.

(a)

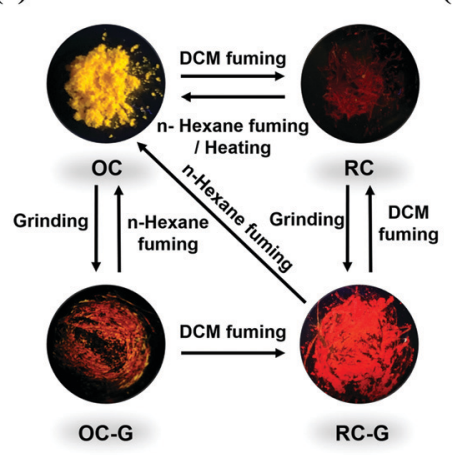

(b)

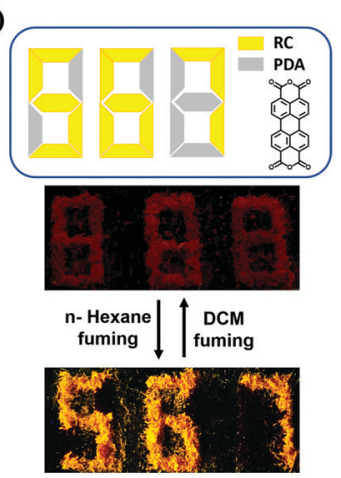

Fig. 7 (a) FL images of the crystal-to-crystal switching processes in response to multiple stimuli. (b) Application of the $\mathbf{R C}$ responsive behavior to solvent vapor in FL encryption: a pattern " 888 " is drawn with RC (yellow) and 3,4,9,10-perylenetetracarboxylic dianhydride (PDA) (gray). The red pattern is reversibly switched to orange pattern "567" upon vapor fuming.

\section{Conclusions}

In summary, we have reported a polymorphic AIEgen material with a solvent regulated crystal-to-crystal switch feature. Two polymorphs, OC and RC, with different emission properties are obtained by slow evaporation in DCM $/ n$-hexane mixtures with different volume ratios. DCM molecules are found in RC. The relationship between molecular stacking and the emission properties is explored. A crystal-to-crystal switch is observed between OC and RC when exposed to $n$-hexane vapor and DCM vapor, respectively. RC is formed in a more compact stacking mode due to the more planar molecular conformation and stronger intermolecular interactions, and thus exhibits remarkably red-shifted emission and weaker FL emission. The real-time monitoring of the in situ fuming process with micrographs and the FL emission suggests that the molecular re-arrangements caused by capture/release of DCM molecules are responsible for the crystal-to-crystal switch. Multiple stimuli-responsive behaviors of the polymorphs are demonstrated, especially their distinct ML properties. A solvent vapor regulated simple pattern for data encryption and security is achieved with RC under UV light irradiation. Our work has provided a strategy to further understand the relationship of molecular stacking and emission properties for crystal-to-crystal switching.

\section{Conflicts of interest}

There are no conflicts to declare.

\section{Acknowledgements}

This work was financially supported by Beijing Natural Science Foundation (2202043), the National Natural Science Foundation of China (21774007), the Fundamental Research Funds for the Central Universities (ZY2024 and PT1811) and Natural Science Foundation of Shaanxi Province, China (No. 2016ZDJC-19).

\section{Notes and references}

1 (a) S. Mo, Q. Meng, S. Wan, Z. Su, H. Yan, B. Z. Tang and M. Yin, Tunable mechanoresponsive self-assembly of an amide-linked dyad with dual sensitivity of photochromism and mechanochromism, Adv. Funct. Mater., 2017, 27, 1701210; (b) W. Li, Q. Huang, Z. Mao, Q. Li, L. Jiang, Z. Xie, R. Xu, Z. Yang, J. Zhao, T. Yu, Y. Zhang, M. P. Aldred and Z. Chi, Alkyl chain introduction: in situ solar-renewable colorful organic mechanoluminescence materials, Angew. Chem., Int. Ed., 2018, 57, 12727-12732; (c) Y. Li, S. Liu, T. Han, H. Zhang, C. Chuah, R. T. K. Kwok, J. W. Y. Lam and B. Z. Tang, Sparks fly when AIE meets with polymers, Mater. Chem. Front., 2019, 3, 2207-2220.

2 (a) Z. Yang, Z. Chi, Z. Mao, Y. Zhang, S. Liu, J. Zhao, M. P. Aldred and Z. Chi, Recent advances in mechanoresponsive luminescence of tetraphenylethylene derivatives with aggregation-induced emission properties, Mater. Chem. Front., 2018, 2, 861-890; (b) L. Tan, S. Mo, B. Fang, W. Cheng and M. Yin, Dual fluorescence switching of a 
Rhodamine 6G-naphthalimide conjugate with high contrast in the solid state, J. Mater. Chem. C, 2018, 6, 10270-10275; (c) B. Fang, M. Chu, L. Tan, P. Li, Y. Hou, Y. Shi, Y. S. Zhao and M. Yin, Near-infrared microlasers from self-assembled Spiropyrane-based microsphercial caps, ACS Appl. Mater. Interfaces, 2019, 11, 38226-38231.

3 (a) J. Mei, N. L. Leung, R. T. Kwok, J. W. Y. Lam and B. Z. Tang, Aggregation-induced emission: together we shine, united we soar!, Chem. Rev., 2015, 115, 11718-11940; (b) Y. Cai, C. Ji, S. Zhang, Z. Su and M. Yin, Synthesis of water-soluble dye-cored poly(amidoamine) dendrimers for long-term live cell imaging, Sci. China Mater., 2018, 61, 1475-1483; (c) Y. Hong, J. W. Y. Lama and B. Z. Tang, Aggregation-induced emission: phenomenon, mechanism and applications, Chem. Commun., 2009, 4332-4353; (d) C. Ji, W. Cheng, Q. Yuan, K. Müllen and M. Yin, From dyestuff chemistry to cancer theranostics: the rise of rylenecarboximides, Acc. Chem. Res., 2019, 52, 2266-2277.

4 (a) J. Luo, Z. Xie, J. W. Y. Lam, L. Cheng, H. Chen, C. Qiu, H. S. Kwok, X. Zhan, Y. Liu, D. Zhu and B. Z. Tang, Aggregation-induced emission of 1-methyl-1,2,3,4,5-pentaphenylsilole, Chem. Commun., 2001, 1740-1741; (b) Y. Hong, J. W. Y. Lam and B. Z. Tang, Aggregation-induced emission, Chem. Soc. Rev., 2011, 40, 5361-5388; (c) J. Huang, N. Sun, J. Yang, R. Tang, Q. Li, D. Ma and Z. Li, Blue aggregationinduced emission luminogens: high external quantum efficiencies up to $3.99 \%$ in LED device, and restriction of the conjugation length through rational molecular design, Adv. Funct. Mater., 2014, 24, 7645-7654; (d) J. Yang, Z. Chi, W. Zhu, B. Z. Tang and Z. Li, Aggregation-induced emission: a coming-of-age ceremony at the age of eighteen, Sci. China: Chem., 2019, 62, 1090-1098.

5 (a) Z. Chi, X. Zhang, B. Xu, X. Zhou, C. Ma, Y. Zhang, S. Liu and J. Xu, Recent advances in organic mechanofluorochromic materials, Chem. Soc. Rev., 2012, 41, 3878-3896; (b) R. Hu, N. L. C. Leung and B. Z. Tang, AIE macromolecules: syntheses, structures and functionalities, Chem. Soc. Rev., 2014, 43, 4494-4562; (c) P. Li, B. Lv, D. Han, P. Duan, M. Liu and M. Yin, Stoichiometry-controlled inversion of circularly polarized luminescence in co-assembly of chiral gelators with an achiral tetraphenylethylene derivative, Chem. Commun., 2019, 55, 2194-2197; (d) J. Yang, L. Li, Y. Yu, Z. Ren, Q. Peng, S. Ye, Q. Li and Z. Li, Blue pyrenebased AIEgens: inhibited intermolecular $\pi-\pi$ stacking through the introduction of substituents with controllable intramolecular conjugation, and high external quantum efficiencies up to $3.46 \%$ in non-doped OLEDs, Mater. Chem. Front., 2017, 1, 91-99.

6 (a) Y. Xie and Z. Li, The development of mechanoluminescence from organic compounds: breakthrough and deep insight, Mater. Chem. Front., 2020, 4, 317-331; (b) L. Yang, P. Ye, W. Li, W. Zhang, Q. Guan, C. Ye, T. Dong, X. Wu, W. Zhao, X. Gu, Q. Peng, B. Z. Tang and H. Huang, Uncommon aggregation-induced emission molecular materials with highly planar conformations, Adv. Opt. Mater., 2018, 6, 1701394; (c) X. Ge, M. Gao, B. Situ, W. Feng, B. He, X. He,
S. Li, Z. Ou, Y. Zhong, Y. Lin, X. Ye, X. Hu, B. Z. Tang and L. Zheng, One-step, rapid fluorescence sensing of fungal viability based on a bioprobe with aggregation-induced emission characteristics, Mater. Chem. Front., 2020, 4, 957-964.

7 (a) C. Ge, J. Liu, X. Ye, Q. Han, L. Zhang, S. Cui, Q. Guo, G. Liu, Y. Liu and X. Tao, Visualization of single-crystal-tosingle-crystal phase transition of luminescent molecular polymorphs, J. Phys. Chem. C, 2018, 122, 15744-15752; (b) S. Varghese, S. K. Park, S. Casado, R. Resel, R. Wannemacher, L. Lüer, S. Y. Park and J. Gierschner, Polymorphism and amplified spontaneous emission in a dicyano-distyrylbenzene derivative with multiple trifluoromethyl substituents: intermolecular interactions in play, Adv. Funct. Mater., 2016, 26, 2349-2356; (c) Q. Li, W. Jin, M. Chu, W. Zhang, J. Gu, B. Shahid, A. Chen, Y. Yu, S. Qiao and Y. S. Zhao, Tailoring the structures and photonic properties of low-dimensional organic materials by crystal engineering, Nanoscale, 2018, 10, 4680-4685; (d) J. Y. Zhu, C. X. Li, P. Chen, Z. Ma, B. Zou, L. Y. Niu, G. Cui and Q. Z. Yang, A polymorphic fluorescent material with strong solid state emission and multi-stimuli-responsive properties, Mater. Chem. Front., 2020, 4, 176-181; (e) Q. Li and Z. Li, Molecular packing: another key point for the performance of organic and polymeric optoelectronic materials, Acc. Chem. Res., 2020, 53, 962-973.

8 (a) B. Fang, M. Chu, Z. Wu, Y. Shi, Y. S. Zhao and M. Yin, Efficient triphenylamine-based polymorphs with different mechanochromism and lasing emission: manipulating molecular packing and intermolecular interactions, J. Mater. Chem. C, 2019, 7, 4434-4440; (b) D. Yan and D. G. Evans, Molecular crystalline materials with tunable luminescent properties: from polymorphs to multi-component solids, Mater. Horiz., 2014, 1, 46-57; (c) D. Yan, G. Fan, Y. Guan, Q. Meng, C. Li and J. Wang, Tuning solid-state blue and red luminescence by the formation of solvate crystals, Phys. Chem. Chem. Phys., 2013, 15, 19845-19852; (d) Z. Xie, T. Yu, J. Chen, E. Ubba, L. Wang, Z. Mao, T. Su, Y. Zhang, M. P. Aldred and Z. Chi, Weak interactions but potent effect: tunable mechanoluminescence by adjusting intermolecular C-H $\cdots \pi$ interactions, Chem. Sci., 2018, 9, 5787-5794.

9 (a) J. Wang, Z. Chai, J. Wang, C. Wang, M. Han, Q. Liao, A. Huang, P. Lin, C. Li, Q. Li and Z. Li, Mechanoluminescence or room-temperature phosphorescence: molecular packingdependent emission response, Angew. Chem., Int. Ed., 2019, 131, 17457-17462; (b) Y. Wang, G. Zhang, W. Zhang, X. Wang, Y. Wu, T. Liang, X. Hao, H. Fu, Y. Zhao and D. Zhang, Tuning the solid state emission of the carbazole and cyano-substituted tetraphenylethylene by co-crystallization with solvents, Small, 2016, 12, 6554-6561; (c) S. Jiang, J. Wang, Q. Qi, J. Qian, B. Xu, F. Li, Q. Zhou and W. Tian, Organic polymorphs with fluorescence switching: direct evidence for mechanical and thermal modulation of excited state transitions, Chem. Commun., 2019, 55, 3749-3752; (d) Q. Liao, X. G. Wang, S. Lv, Z. Xu, Y. Zhang and $\mathrm{H}$. Fu, Cluster-mediated nucleation and growth of $\mathrm{J}$ - and $\mathrm{H}$-type polymorphs of difluoroboron avobenzone for organic microribbon lasers, ACS Nano, 2018, 12, 5359-5367. 
10 X. Cheng, Z. Wang, B. Tang, H. Zhang, A. Qin, J. Z. Sun and B. Z. Tang, Diversified photo/electronic functions based on a simple chalcone skeleton: effects of substitution pattern and molecular packing, Adv. Funct. Mater., 2018, 28, 1706506.

11 P. Z. Chen, H. Zhang, L. Y. Niu, Y. Zhang, Y. Z. Chen, H. Fu and Q. Z. Yang, A solid-state fluorescent material based on carbazole-containing difluoroboron $\beta$-diketonate: multiple chromisms, the self-Assembly behavior, and optical waveguides, Adv. Funct. Mater., 2017, 27, 1700332.

12 (a) K. Ling, H. Shi, H. Wang, L. Fu, A. Lv, K. Huang, W. Ye, M. Gu, C. Ma, X. Yao, W. Jia, J. Zhi, W. Yao, Z. An, H. Ma and W. Huang, Controllable multiemission with ultralong organic phosphorescence in crystal by isomerization, $A d v$. Opt. Mater., 2019, 7, 1901076; (b) Z. He, H. Gao, S. Zhang, S. Zheng, Y. Wang, Z. Zhao, D. Ding, B. Yang, Y. Zhang and W. Z. Yuan, Achieving persistent, efficient, and robust room-temperature phosphorescence from pure organics for versatile applications, Adv. Mater., 2019, 31, 1807222.

13 B. Huang, W. C. Chen, Z. Li, J. Zhang, W. Zhao, Y. Feng, B. Z. Tang and C. S. Lee, Manipulation of molecular aggregation states to realize polymorphism, AIE, MCL, and TADF in a single molecule, Angew. Chem., Int. Ed., 2018, 130, 12653-12657.

14 (a) X. Yang, Q. Wang, P. Hu, C. Xu, W. Guo, Z. Wang, Z. Mao, Z. Yang, C. Liu, G. Shi, L. Chen, B. Xu and Z. Chi, Achieving remarkable and reversible mechanochromism from bright ionic AIEgen with high specificity for mitochondria imaging and secondary aggregation emission enhancement for longterm tracking of tumor, Mater. Chem. Front., 2020, 4, 941-949; (b) C. Wang, Y. Yu, Z. Chai, F. He, C. Wu, Y. Gong, M. Han, Q. Li and Z. Li, Recyclable mechanoluminescent luminogen: different polymorphs, different selfassembly effects of the thiophene moiety and recovered molecular packing via simple thermal-treatment, Mater. Chem. Front., 2019, 3, 32-38; (c) H. Sun, S. S. Sun, F. F. Han, Z. H. Ni, R. Zhang and M. D. Li, A new tetraphenylethene-based Schiff base: two crystalline polymorphs exhibiting totally different photochromic and fluorescence properties, J. Mater. Chem. C, 2019, 7, 7053-7064; (d) B. Xu, J. He, Y. Mu, Q. Zhu, S. Wu, Y. Wang, Y. Zhang, C. Jin, C. Lo, Z. Chi, A. Lien, S. Liu and $\mathrm{J}$. $\mathrm{Xu}$, Very bright mechanoluminescence and remarkable mechanochromism using a tetraphenylethene derivative with aggregation-induced emission, Chem. Sci., 2015, 6, 3236-3241.
15 (a) H. Ma, H. Yu, Q. Peng, Z. An, D. Wang and Z. Shuai, Hydrogen bonding-induced morphology dependence of long-lived organic room-temperature phosphorescence: a computational study, J. Phys. Chem. Lett., 2019, 10, 6948-6954; (b) S. Yang, P. A. Yin, L. Li, Q. Peng, X. Gu, G. Gao, J. You and B. Z. Tang, Crystallization-induced reversal from dark to bright excited states for construction of solid-emission-tunable squaraines, Angew. Chem., Int. Ed., 2020, DOI: 10.1002/anie.201914437.

16 (a) X. Cheng, F. Yang, J. Zhao, J. Ni, X. He, C. Zhou, J. Z. Sun and B. Z. Tang, Microscopic visualization and mechanism investigation on crystal jumping behavior of a cyclic chalcone derivative, Mater. Chem. Front., 2020, 4, 651-660; (b) Y. Yu, Y. Fan, C. Wang, Y. Wei, Q. Liao, Q. Li and Z. Li, Phenanthroimidazole derivatives with minor structural differences: crystalline polymorphisms, different molecular packing, and totally different mechanoluminescence, J. Mater. Chem. C, 2019, 7, 13759-13763.

17 (a) W. Yang, C. Liu, S. Lu, J. Du, Q. Gao, R. Zhang, Y. Liu and C. Yang, AIE-active smart cyanostyrene luminogens: polymorphism-dependent multicolor mechanochromism, J. Mater. Chem. C, 2018, 6, 290-298; (b) C. Wang and Z. Li, Molecular conformation and packing: their critical roles in the emission performance of mechanochromic fluorescence materials, Mater. Chem. Front., 2017, 1, 2174-2194; (c) C. Wang, B. Xu, M. Li, Z. Chi, Y. Xie, Q. Li and Z. Li, A stable tetraphenylethene derivative: aggregation-induced emission, different crystalline polymorphs, and totally different mechanoluminescence properties, Mater. Horiz., 2016, 3, 220-225; (d) S. Li and D. Yan, Tuning light-driven motion and bending in macroscale-flexible molecular crystals based on a cocrystal approach, ACS Appl. Mater. Interfaces, 2018, 10, 22703-22710.

18 (a) J. Yang, J. Qin, P. Geng, J. Wang, M. Fang and Z. Li, Molecular conformation-dependent mechanoluminescence: same mechanical stimulus but different emissive color over time, Angew. Chem., Int. Ed., 2018, 130, 14370-14374; (b) Z. Wu, S. Mo, L. Tan, B. Fang, Z. Su, Y. Zhang and M. Yin, Crystallization-induced emission enhancement of a deep-blue luminescence material with tunable mechano- and thermochromism, Small, 2018, 14, 1802524; (c) B. Shao, R. Jin, A. Li, Y. Liu, B. Li, S. Xu, W. Xu, B. Xu and W. Tian, Luminescent switching and structural transition through multiple external stimuli based on organic molecular polymorphs, J. Mater. Chem. C, 2019, 7, 3263-3268. 